\title{
THE FACTORIAL MOMENTS OF ADDITIVE FUNCTIONS WITH RATIONAL ARGUMENT
}

\author{
J. ŠIAULYS and G. STEPANAUSKAS ${ }^{\bowtie}$
}

(Received 20 July 2004; revised 24 May 2005)

Communicated by W. W. L. Chen

\begin{abstract}
We consider the weak convergence of the set of strongly additive functions $f(q)$ with rational argument $q$. It is assumed that $f(p)$ and $f(1 / p) \in\{0,1\}$ for all primes. We obtain necessary and sufficient conditions of the convergence to the limit distribution. The proof is based on the method of factorial moments. Sieve results, and Halász's and Ruzsa's inequalities are used. We present a few examples of application of the given results to some sets of fractions.
\end{abstract}

2000 Mathematics subject classification: primary $11 \mathrm{~K} 65,11 \mathrm{~N} 37,11 \mathrm{~N} 64$.

Keywords and phrases: additive arithmetic function, factorial moment, limit distribution, Farey fraction.

\section{Introduction}

Let $\left\{f_{x}: \mathbb{N} \rightarrow \mathbb{R}, x \geq 2\right\}$ be a set of additive functions, $A$ be some subset of natural numbers, and

$$
v_{x}(A):=\frac{1}{[x]} \#\{n \leq x, n \in A\}
$$

be a frequency of natural numbers $n \in A$. The set $A$ is allowed to depend on $x$ and other parameters.

The central problem of probabilistic number theory is to find conditions under which the frequencies $v_{x}\left(f_{x}(n)-\alpha(x)<u\right)$, with a suitably chosen centering function $\alpha(x)$, converge to the limit law as $x \rightarrow \infty$. Starting with Turán's proof of the HardyRamanujan theorem on the normal order of prime factors in 1934, many works have been devoted to this problem. This problem is the main object of the monographs of

(C) 2006 Australian Mathematical Society $1446-7887 / 06 \$ A 2.00+0.00$ 
Kubilius [5] and Elliott $[1,2]$. There are three different cases in the investigation of a set of additive functions (see, for example, $[1,2,5])$. The first case is when $f_{x}(n)$ does not depend on $x$. The Erdös-Wintner theorem (see, for example, [2, page 187]) is the most celebrated result of this case. The second case arises when $f_{x}(n)=f(n) / b(x)$, where $b(x)$ is some normalizing unbounded function satisfying some additional conditions. The well-known Levin-Timofeev theorem (see, for example, [2, pages 122-123]) is one such result about the weak convergence of distributions $v_{x}(f(n) / b(x)-\alpha(x)<u)$ to the limit law.

The third, most general case, we obtain when the additive function $f_{x}$ depends on $x$ in an arbitrary way. The first result in this direction was obtained by Rusza. He found necessary and sufficient conditions for the weak convergence of $v_{x}\left(f_{x}(n)-\alpha(x)<u\right)$ to the improper law (see [6]). Šiaulys continued the investigation of the set of such functions. He derived necessary and sufficient conditions for the convergence of the distributions $v_{x}\left(f_{x}(n)<u\right)$ to the Poisson law in the case when $f_{x}$ are strongly additive and $f_{x}(p) \in\{0,1\}$ (see [8]).

In the present paper we consider the additive function defined on the set of positive rational numbers $\mathbb{Q}_{+}$. We suppose throughout that the natural numbers in the representation of the rational number $q=m / n$, are coprime, that is, $(m, n)=1$. Any rational number $q$ has a unique representation as a product $q=p_{1}^{\alpha_{1}} \cdots p_{s}^{\alpha_{s}}$, where $p_{1}, \ldots, p_{s}$ are distinct prime numbers, and $\alpha_{1}, \ldots, \alpha_{s}$ are integers. The power of prime $p$ in such a product for the rational $q$ is denoted by $\alpha_{p}(q)$. We say that the rational number $q_{1}=m_{1} / n_{1}$ divides $q_{2}=m_{2} / n_{2}\left(q_{1} \mid q_{2}\right)$ if $m_{1} \mid m_{2}$ and $n_{1} \mid n_{2}$, and that they are coprime if $\left(m_{1}, m_{2}\right)=\left(m_{1}, n_{2}\right)=\left(m_{2}, n_{1}\right)=\left(n_{1}, n_{2}\right)=1$.

For any additive function $f: \mathbb{Q}_{+} \rightarrow \mathbb{C}$ the equality $f(q)=\sum_{p} f\left(p^{\alpha_{p}(q)}\right)$ holds. If, in addition, $f\left(p^{\alpha}\right)=f\left(p^{\mathrm{sgn} \alpha}\right)$ for all integers $\alpha$ and primes $p$, the function $f$ is called strongly additive. Thus for any strongly additive function $f$ with rational argument $f(q)=\sum_{p^{\delta} \mid q} f\left(p^{\delta}\right)$, where $\delta \in\{-1,1\}$.

For $x \geq 2$, for an interval $I:=(\xi, \eta]$, and for some condition $A$, where $\xi, \eta$ and $A$ are allowed to depend on $x$, we write:

$$
\begin{aligned}
\mathbb{Q}_{x}^{I} & :=\left\{q=\frac{m}{n}: n \leq x, \xi<\frac{m}{n} \leq \eta\right\}, & \mathbb{E}_{x}^{I}\left(p^{\alpha}\right):=\left\{q \in \mathbb{Q}_{x}^{I}: \alpha_{p}(q)=\alpha\right\}, \\
v_{x}^{I}(A) & :=\left(\# \mathbb{Q}_{x}^{I}\right)^{-1} \#\left\{q \in \mathbb{Q}_{x}^{I}: q \in A\right\}, & \mathbb{P}_{x}^{I}:=\left\{p^{\operatorname{sgn} \alpha}: \mathbb{E}_{x}^{I}\left(p^{\alpha}\right) \neq \emptyset\right\} .
\end{aligned}
$$

In the expression for $v_{x}^{I}(A)$ we suppose $\# \mathbb{Q}_{x}^{I}>0$. We call the elements from $\mathbb{P}_{x}^{I}$ the prime ones. The quantity $v_{x}^{l}(A)$ denotes the frequency of the rational numbers which satisfy the condition $A$. In the particular case $I=(0,1]$ we omit the symbol $I$ and instead of $\mathbb{Q}_{x}^{I}, \mathbb{E}_{x}^{l}\left(p^{\alpha}\right), \mathbb{P}_{x}^{I}, v_{x}^{I}(A)$ we simply write $\mathbb{Q}_{x}, \mathbb{E}_{x}\left(p^{\alpha}\right), \mathbb{P}_{x}, v_{x}(A)$. We observe that $\mathbb{Q}_{x}=\{m / n: n \leq x, m / n \leq 1\}$ is the classical set of Farey fractions.

The probabilistic model for solving problems on the value distribution of additive functions with rational arguments can be developed in analogy with the Kubilius 
model (see $[4,11,13,12])$. In this work, we consider the distribution of the set of strongly additive functions $v_{x}\left(f_{x}(q)<u\right)$ using the factorial moments method.

Throughout the paper we use the following notations. The function $\varepsilon(x)$ is always vanishing as $x$ tends to infinity. The absolute constants are denoted by $c_{1}, c_{2}, \ldots$ The expression $a \ll b$ is equivalent to $|a| \leq c b$, with some positive constant $c$. If the vanishing function or bounding quantities depend on $d$, we write $\varepsilon_{d}(x), O_{d}, \ll_{d}$.

Let $\widetilde{\mathbb{P}}_{x}:=\left\{p^{\delta} \in \mathbb{P}_{x}: f_{x}\left(p^{\delta}\right)=1\right\}$. For the sake of brevity, we use a star and a $t$ above the summation sign $\sum^{* t}$ to denote a summation expanded over all collections of $p_{1}^{\delta_{1}}, p_{2}^{\delta_{2}}, \ldots, p_{t}^{\delta_{t}} \in \widetilde{\mathbb{P}}_{x}$ (with some fixed $t$ ) such that $p_{i} \neq p_{j}, 1 \leq i<j \leq t$. The most frequent case is when $t=l$. In this case we will omit $l$ and write $\sum^{*}$.

The main result of this paper is the following statement.

THEOREM 1.1. Let $\left\{f_{x}, x \geq 2\right\}$ be a set of strongly additive functions with rational argument. Let $f_{x}\left(p^{\delta}\right) \in\{0,1\}$ for every prime number $p$ and exponent $\delta \in\{-1,1\}$. Then the frequency $v_{x}\left(f_{x}(q)<u\right)$ converges weakly to some distribution function if and only if the limit

$$
\lim _{x \rightarrow \infty} \sum^{*} \frac{\Delta_{x}\left(p_{1}^{\delta_{1}} p_{2}^{\delta_{2}} \cdots p_{l}^{\delta_{l}}\right)}{\left(p_{1}+1\right)\left(p_{2}+1\right) \cdots\left(p_{l}+1\right)}=g_{l}
$$

exists for every fixed natural number 1 . Here

$$
\Delta_{x}(q):= \begin{cases}1 & \text { if it exists } q_{1} \in \mathbb{Q}_{x}: q \mid q_{1}, \\ 0 & \text { if that } q_{1} \text { does not exist. }\end{cases}
$$

Moreover, if the limit distribution exists, then its characteristic function is equal to $1+\sum_{l=1}^{\infty}\left(g_{l} / l !\right)\left(\mathrm{e}^{i t}-1\right)^{l}$.

\section{Examples}

Using Theorem 1.1 we can calculate the asymptotic densities of some arithmetically interesting sets of fractions. Let us give a few examples.

EXAMPLE 1. Define the strongly additive function $f$ by

$$
f(p)=\left\{\begin{array}{ll}
1 & \text { if } p=2,3, \\
0 & \text { otherwise, }
\end{array} \text { and } f(1 / p)= \begin{cases}1 & \text { if } p=3,5 \\
0 & \text { otherwise }\end{cases}\right.
$$

It follows from (1.1) that $g_{1}=1, g_{2}=11 / 18, g_{3}=1 / 6, g_{l}=0, l \geq 4$. Hence the limit law of $v_{x}(f(q)<u)$ has the characteristic function

$$
\frac{5}{18}+\frac{17}{36} \mathrm{e}^{i t}+\frac{2}{9} \mathrm{e}^{2 i t}+\frac{1}{36} \mathrm{e}^{3 i t}
$$


From the well-known asymptotics $\#\left\{q \in \mathbb{Q}_{x}\right\} \sim\left(3 / \pi^{2}\right) x^{2}, x \rightarrow \infty$, and the structure of characteristic function (2.1), we have that

$\#\left\{q \in \mathbb{Q}_{x}:\right.$ prime elements $2,3,1 / 3,1 / 5$ do not divide $\left.q\right\} \sim \frac{5}{6 \pi^{2}} x^{2}$,

$\#\left\{q \in \mathbb{Q}_{x}:\right.$ exactly two elements from $\{2,3,1 / 3,1 / 5\}$ divide $\left.q\right\} \sim \frac{2}{3 \pi^{2}} x^{2}$,

$\#\left\{q \in \mathbb{Q}_{x}:\right.$ exactly three elements from $\{2,3,1 / 3,1 / 5\}$ divide $\left.q\right\} \sim \frac{1}{12 \pi^{2}} x^{2}$, as $x \rightarrow \infty$.

EXAMPLE 2. Define the strongly additive function $f_{x}$ by

$$
f_{x}(p)=f_{x}(1 / p)= \begin{cases}1 & \text { if } \log x<p \leq \log ^{2} x, \\ 0 & \text { otherwise. }\end{cases}
$$

In this case $g_{1}=2 \log 2, g_{2}=(2 \log 2)^{2}, g_{l}=(2 \log 2)^{l}, l \geq 3$. Thus the limit law of $v_{x}\left(f_{x}(q)<u\right)$ is the Poisson law with parameter $\lambda=2 \log 2$, and we have $\#\left\{q \in \mathbb{Q}_{x}: p \nmid q,(1 / p) \nmid q\right.$ for $\left.p \in\left(\log x, \log ^{2} x\right]\right\} \sim \frac{3}{4 \pi^{2}} x^{2}$, $\#\left\{q \in \mathbb{Q}_{x}: q\right.$ has exactly one prime divisor $p^{\delta}$ for $\left.p \in\left(\log x, \log ^{2} x\right]\right\} \sim \frac{3 \log 2}{2 \pi^{2}} x^{2}$, $\#\left\{q \in \mathbb{Q}_{x}: q\right.$ has exactly two prime divisors $p^{\delta}$ for $\left.p \in\left(\log x, \log ^{2} x\right]\right\} \sim \frac{3 \log ^{2} 2}{2 \pi^{2}} x^{2}$. as $x \rightarrow \infty$.

EXAMPLE 3. Let the strongly additive function $f_{x}$ be defined by

$$
f_{x}(p)=f_{x}(1 / p)= \begin{cases}1 & \text { if } \sqrt[3]{x}<p \leq x \\ 0 & \text { otherwise }\end{cases}
$$

Put $\theta=\log 3 \log 2+\mathrm{Li}_{2}(1 / 3)-\mathrm{Li}_{2}(2 / 3)$, where $\mathrm{Li}_{2}(u)$ is the polylogarithm of second order, that is,

$$
\mathrm{Li}_{2}(u)=\sum_{k=1}^{\infty} \frac{u^{k}}{k^{2}}, \quad|u| \leq 1 .
$$

We have that $g_{1}=2 \log 3, g_{2}=2\left((\log 3)^{2}+\theta\right), g_{3}=6 \theta \log 3, g_{4}=6 \theta^{2}, g_{l}=0$, $l \geq 5$.

Since the limit law of $v_{x}\left(f_{x}(q)<u\right)$ has the characteristic function

$$
\begin{gathered}
(1-\log 3)(1-\log 3+\theta)+\theta^{2} / 4+(2 \log 3(1-\log 3)+(3 \log 3-2-\theta) \theta) \mathrm{e}^{i t} \\
+\left(\log ^{2} 3+(1-3 \log 3+3 \theta / 2) \theta\right) \mathrm{e}^{2 i t}+(\log 3-\theta) \theta \mathrm{e}^{3 i t}+\theta^{2} \mathrm{e}^{4 i t} / 4,
\end{gathered}
$$


the following asymptotic expressions (for $x \rightarrow \infty$ ) are true:

$$
\begin{aligned}
& \#\left\{q \in \mathbb{Q}_{x}: \text { prime elements from }\{p, 1 / p, p \in(\sqrt[3]{x}, x]\} \text { do not divide } q\right\} \\
& \sim \frac{3}{\pi^{2}}\left((1-\log 3)(1-\log 3+\theta)+\theta^{2} / 4\right) x^{2} \approx 0.00072 x^{2}
\end{aligned}
$$

\section{Auxiliary lemmas}

The proof of Theorem 1.1 is based on the factorial moments method, but some sieve results (Lemma 3.1), and the inequalities of Halász (see [3]) and Ruzsa (see [7]) are of key importance as well. In this section we present the analogues, sufficient for our needs, of these inequalities for functions of rational argument (Lemma 3.2 and Lemma 3.3).

LEMMA 3.1 (see [12]). Let $I=(\xi, \eta], 0 \leq \xi<\eta$, be an interval of real numbers. Let $N_{0}, N_{1}, N_{2}$ be natural numbers, which do not have any common prime divisor. All quantities $\xi, \eta, N_{0}, N_{1}, N_{2}$ may depend on $x \geq 2$. Then

$$
\begin{aligned}
\#\left\{\frac{m}{n} \in\right. & \left.\mathbb{Q}_{x}^{I}:\left(m, N_{0} N_{1}\right)=\left(n, N_{0} N_{2}\right)=1\right\} \\
= & \frac{3}{\pi^{2}}(\eta-\xi) x^{2} \prod_{p \mid N_{0}}\left(1-\frac{2}{p+1}\right) \prod_{p \mid N_{1} N_{2}}\left(1-\frac{1}{p+1}\right) \\
& \times\left(1+2^{\omega\left(N_{0} N_{1} N_{2}\right)} O\left(\frac{\log x}{x}+\frac{1}{x(\eta-\xi)}\right)\right),
\end{aligned}
$$

where $\omega(m)$ is the number of distinct primes dividing $m$.

LEMMA 3.2 (see [10]). Let $I=(\xi, \eta]$ be an interval of real numbers, where $\xi$ and $\eta$ may depend on $x \geq 2$ and satisfy the limit conditions:

$$
\limsup _{x \rightarrow \infty} \frac{\xi}{x(\eta-\xi)} \leq c_{1}, \quad \lim _{x \rightarrow \infty} \frac{1}{x(\eta-\xi)}=0 .
$$


Let $f: \mathbb{Q} \rightarrow \mathbb{N} \cup\{0\}$ be an integer-valued additive function. Then, for every $L \in \mathbb{N} \cup\{0\}$,

$$
v_{x}^{I}(f(q)=L) \leq c_{2}\left(\max \left\{\sum_{p \in \mathbb{P}_{x}^{\prime}, f(p) \neq 0} 1 / p, \sum_{(1 / p) \in \mathbb{P}_{x}^{\prime}, f(1 / p) \neq 0} 1 / p\right\}\right)^{-1 / 2},
$$

where $c_{2}$ depends on $c_{1}$ and on the convergence rate in (3.1).

LEMMA 3.3 (see [9]). Let $I=(\xi, \eta]$ be an interval satisfying the conditions $\xi \leq c_{3} x(\eta-\xi), x(\eta-\xi) \geq c_{4}$. Then, for an arbitrary strongly additive function $f: \mathbb{Q} \rightarrow \mathbb{C}$ and for every natural number $l$,

$$
\sum_{q \in \mathbb{Q}_{x}^{\prime}}\left|f(q)-\sum_{p^{\delta} \in \mathbb{P}_{x}^{\prime}} \frac{f\left(p^{\delta}\right)}{p}\right|^{l} \ll x^{2}(\eta-\xi)\left(\left(\sum_{p^{\delta} \in \mathbb{P}_{x}^{l}} \frac{\left|f\left(p^{\delta}\right)\right|^{2}}{p}\right)^{l / 2}+\sum_{p^{\delta} \in \mathbb{P}_{x}^{\prime}} \frac{\left|f\left(p^{\delta}\right)\right|^{l}}{p}\right),
$$

where the constant implied in the symbol $\ll$ may depend on $c_{3}, c_{4}$ and $l$.

\section{Boundedness of factorial moments}

PROPOSITION 4.1. Let $I=(\xi, \eta]$ be an interval of real numbers, where $\xi$ and $\eta$ may depend on $x \geq 2$ and satisfy the limit conditions:

$$
\limsup _{x \rightarrow \infty} \frac{\xi}{x(\eta-\xi)}<\infty, \quad \lim _{x \rightarrow \infty} \frac{1}{x(\eta-\xi)}=0 .
$$

Let $f_{x}$ be a set of strongly additive functions with rational argument. Let $f_{x}\left(p^{\delta}\right) \in$ $\{0,1\}$ for every prime number $p$ and exponent $\delta \in\{-1,1\}$. Let

$$
\varphi(l, x)=\frac{1}{\# \mathbb{Q}_{x}^{l}} \sum_{q \in \mathbf{Q}_{x}^{\prime}} f_{x}(q)\left(f_{x}(q)-1\right) \cdots\left(f_{x}(q)-l+1\right)
$$

for every natural number $l$.

If the distributions $v_{x}^{I}\left(f_{x}(q)<u\right)$ have a weak limit as $x \rightarrow \infty$, then

$$
\limsup _{x \rightarrow \infty} \varphi(l, x) \ll 1 .
$$

Here the constant in $\ll$ depends on $l$ and on the structure of the limit law.

PRoof. Suppose $X$ is a random variable for which

$$
v_{x}^{I}\left(f_{x}(q)<u\right) \underset{x \rightarrow \infty}{\longrightarrow} P(X<u) .
$$


The random variable $X$ is integer valued, hence there exists $L \in\{0\} \cup \mathbb{N}$ for which $P(X=L)>0$. From the limit

$$
\lim _{x \rightarrow \infty} v_{x}^{l}\left(f_{x}(q)=L\right)=P(X=L),
$$

we have that $v_{x}^{l}\left(f_{x}(q)=L\right) \geq \frac{1}{2} P(X=L)$ for $x \geq c_{5}$, where $c_{5}$ depends on $P(X=L)$ and on the rate of convergence in (4.2).

It follows from Lemma 3.2 that

$$
\max \left\{\sum_{p \in \mathbb{P}_{x}^{\prime}, f_{x}(p)=1} 1 / p, \sum_{1 / p \in \mathbb{P}_{x}^{\prime}, f_{x}(1 / p)=1} 1 / p\right\} \ll \frac{1}{P^{2}(X=L)} .
$$

Hence

$$
\limsup _{x \rightarrow \infty} \sum_{p^{s} \in \mathbb{P}_{x}^{\prime}, f_{x}\left(p^{s}\right)=1} 1 / p \leq c_{6} .
$$

The constant $c_{6}$ depends on the structure of the limit random variable. According to Lemma $3.1, \# \mathbb{Q}_{x}^{I} \gg x^{2}(\eta-\xi)$ for $x$ sufficiently large. Therefore from (4.3) and Lemma 3.3 we obtain

$$
\limsup _{x \rightarrow \infty} \frac{1}{\# \mathbb{Q}_{x}^{l}} \sum_{q \in \mathbf{Q}_{x}^{\prime}}\left|f_{x}(q)-\sum_{p^{\delta} \in \mathbb{P}_{x}^{\prime}} \frac{f_{x}\left(p^{\delta}\right)}{p}\right|^{l} \ll_{l}\left(c_{6}^{l / 2}+c_{6}\right) .
$$

Since

$$
\sum_{q \in \mathbf{Q}_{x}^{\prime}} f_{x}^{l}(q) \leq 2^{l}\left(\sum_{q \in \mathbf{Q}_{x}^{l}}\left|f_{x}(q)-\sum_{p^{\delta} \in \mathbb{P}_{x}^{\prime}} \frac{f_{x}\left(p^{\delta}\right)}{p}\right|^{l}+\# \mathbb{Q}_{x}^{l}\left(\sum_{p^{\delta} \in \mathbb{P}_{x}^{\prime}} \frac{f_{x}\left(p^{\delta}\right)}{p}\right)^{l}\right),
$$

we conclude finally from (4.3) that

$$
\limsup _{x \rightarrow \infty} \varphi(l, x) \leq \limsup _{x \rightarrow \infty} \frac{1}{\# \mathbb{Q}_{x}^{l}} \sum_{q \in \mathbb{Q}_{x}^{l}} f_{x}^{l}(q) \ll_{l} \max \left(c_{6}^{l / 2}, 1\right) .
$$

Proposition 4.1 is proved.

\section{The factorial moments method}

PROPOSITION 5.1. Let $I=(\xi, \eta)$ be an interval of real numbers and $f_{x}$ be a set of strongly additive functions satisfying the condition of Proposition 4.1. Let $X$ be an integer-valued random variable and

$$
g_{l}=\sum_{k=l}^{\infty} k(k-1) \cdots(k-l+1) P(X=k)
$$


for every natural l. If $g_{l+2}<\infty$ for some $l$ and

$$
v_{x}^{I}\left(f_{x}(q)<u\right) \underset{x \rightarrow \infty}{\Longrightarrow} P(X<u),
$$

then

$$
\lim _{x \rightarrow \infty} \varphi(l, x)=g_{l} .
$$

Furthermore, if (5.2) is satisfied for every fixed natural $l$ and $\sum_{l=1}^{\infty}\left(2^{l} g_{l} / l !\right)<\infty$, then (5.1) holds for a random variable $X$ that has the characteristic function

$$
1+\sum_{l=1}^{\infty} \frac{g_{l}}{l !}\left(e^{i t}-1\right)^{l}
$$

ProOF (necessity). Let condition (5.1) be satisfied. Since the random variable $X$ is integer-valued, it follows from (5.1) that

$$
v_{x}^{I}\left(f_{x}(q)=k\right)=P(X=k)+\varepsilon_{k}(x)
$$

for each fixed $k=0,1,2, \ldots$.

Let us split the factorial moment $\varphi(l, x)$ (see (4.1)) into two parts:

$$
\varphi(l, x)=\beta_{1}(l, x, y)+\beta_{2}(l, x, y), \quad y \geq l+3,
$$

where $\beta_{1}(l, x, y)$ is that part of sum (4.1) for which $f_{x}(q)<[y]$ and $\beta_{2}(l, x, y)$ is the part for which $f_{x}(q) \geq[y]$.

From (5.3) we have

$$
\begin{aligned}
\beta_{1}(l, x, y) & =\sum_{k=l}^{[y]-1} k(k-1) \cdots(k-l+1) \nu_{x}^{I}\left(f_{x}(q)=k\right) \\
& =\sum_{k=l}^{[y]-1} k(k-1) \cdots(k-l+1)\left(P(X=k)+\varepsilon_{k}(x)\right) \\
& =g_{l}+\varepsilon_{y}(x)-\sum_{k=[y]}^{\infty} k(k-1) \cdots(k-l+1) P(X=k) .
\end{aligned}
$$

Since

$$
\begin{aligned}
\sum_{k=[y]}^{\infty} k(k-1) \cdots(k-l+1) P(X=k) \\
\leq \\
\quad \sum_{k=[y]}^{\infty} k(k-1) \cdots(k-l+1) \\
\quad \times \sum_{j=k}^{\infty} \frac{j(j-1) \cdots(j-l+1)(j-l)(j-l-1)}{j(j-1) \cdots(j-l+1)(j-l)(j-l-1)} P(X=j)
\end{aligned}
$$


[9]

$$
\begin{aligned}
\leq & \sum_{k=[y]}^{\infty} \frac{k(k-1) \cdots(k-l+1)}{k(k-1) \cdots(k-l+1)(k-l)(k-l-1)} \\
& \times \sum_{j=l+2}^{\infty} j(j-1) \cdots(j-l-1) P(X=j) \\
\leq & g_{l+2} \sum_{k=[y]}^{\infty} \frac{1}{(k-l-1)^{2}}=g_{l+2} \sum_{r=[y]-l-1}^{\infty} \frac{1}{r^{2}} \leq \frac{g_{l+2}}{[y]-l-2},
\end{aligned}
$$

we find that

$$
\beta_{1}(l, x, y)=g_{l}+\varepsilon_{y}(x)+O\left(\frac{g_{l+2}}{[y]-l-2}\right) .
$$

Applying the estimate of Proposition 4.1 we obtain, for $x$ sufficiently large,

$$
\begin{aligned}
\beta_{2}(l, x, y) & =\frac{1}{\# \mathbb{Q}_{x}^{I}} \sum_{\substack{q \in \mathbb{Q}_{x}^{\prime} \\
f_{x}(q) \geq[y]}} f_{x}(q)\left(f_{x}(q)-1\right) \cdots\left(f_{x}(q)-l+1\right) \frac{f_{x}(q)-l}{f_{x}(q)-l} \\
& \leq \frac{\varphi(l+1, x)}{[y]-l} \ll_{l} \frac{1}{[y]-l} .
\end{aligned}
$$

The last estimate and equalities (5.4) and (5.5) imply that

$$
\varphi(l, x)=g_{l}+\varepsilon_{y}(x)+o\left(\frac{g_{l+2}}{[y]-l-2}\right)+O_{l}\left(\frac{1}{[y]-l}\right) .
$$

Consequently equality (5.2) holds.

PROOF (sufficiency). Let equation (5.2) be satisfied for every fixed natural $l$. Let

$$
\psi_{x}(t)=\frac{1}{\# \mathbb{Q}_{x}^{l}} \sum_{q \in \mathbf{Q}_{x}^{l}} \mathrm{e}^{i t f_{x}(q)}
$$

be the characteristic function of the distribution $v_{x}^{l}\left(f_{x}(q)<u\right)$.

For every $r \in\{0\} \cup \mathbb{N}$ and $L \in \mathbb{N}$

$$
\left|\mathrm{e}^{i t r}-1-\sum_{l=1}^{L}\left(\begin{array}{l}
r \\
l
\end{array}\right)\left(\mathrm{e}^{i t}-1\right)^{l}\right| \leq\left(\begin{array}{c}
r \\
L+1
\end{array}\right)\left|\mathrm{e}^{i t}-1\right|^{L+1} .
$$

Consequently

$$
\psi_{x}(t)=1+\sum_{l=1}^{L} \frac{\varphi(l, x)}{l !}\left(\mathrm{e}^{i t}-1\right)^{l}+O\left(\frac{\varphi(L+1, x)}{(L+1) !}\left|\mathrm{e}^{i t}-1\right|^{L+1}\right)
$$


for every natural number $L$.

According to equality (5.2) we have

$$
\begin{aligned}
\lim _{x \rightarrow \infty} \psi_{x}(t) & =1+\sum_{l=1}^{L} \frac{g_{l}}{l !}\left(\mathrm{e}^{i t}-1\right)^{l}+O\left(\frac{g_{L+1}}{(L+1) !}\left|\mathrm{e}^{i t}-1\right|^{L+1}\right) \\
& =1+\sum_{l=1}^{\infty} \frac{g_{l}\left(\mathrm{e}^{i t}-1\right)^{l}}{l !}+O\left(\sum_{l=L+1}^{\infty} \frac{2^{l} g_{l}}{l !}+\frac{2^{L+1} g_{L+1}}{(L+1) !}\right),
\end{aligned}
$$

where $i \in \mathbb{R}$.

Letting $L$ go to infinity, we can assert that

$$
\lim _{x \rightarrow \infty} \psi_{x}(t)=1+\sum_{l=1}^{\infty} \frac{g_{l}}{l !}\left(\mathrm{e}^{i t}-1\right)^{l}
$$

for every $t \in \mathbb{R}$.

Since the limit function is continuous, (5.1) holds for some random variable $X$, which has the above characteristic function. Proposition 5.1 is proved.

\section{Main term of the factorial moment}

PROPOSITION 6.1. Let $f_{x}$ be a set of strongly additive functions with rational argument. Assume $f_{x}\left(p^{\delta}\right) \in\{0,1\}$ for each prime number $p$ and exponent $\delta \in\{-1,1\}$. Then

$$
\varphi(1, x)=\sum_{\substack{p^{\delta} \in \mathbb{P}_{x} \\ f_{x}\left(p^{\delta}\right)=1}} \frac{1}{p+1}+\varepsilon(x)
$$

If

$$
\sum_{\substack{p^{s} \in \mathbb{P}_{x} \\ f_{x}\left(p^{\delta}\right)=1}} \frac{1}{p} \ll 1
$$

then for every natural $l$

$$
\varphi(l, x)=\sum^{*} \frac{\Delta_{x}\left(p_{1}^{\delta_{1}} p_{2}^{\delta_{2}} \cdots p_{l}^{\delta_{l}}\right)}{\left(p_{1}+1\right)\left(p_{2}+1\right) \cdots\left(p_{l}+1\right)}+\varepsilon_{l}(x) .
$$

ProOF. First we consider the case $l=1$. It is evident that

$$
\varphi(1, x)=\frac{1}{\# \mathbb{Q}_{x}} \sum_{q \in \mathbb{Q}_{x}} f_{x}(q)=\sum_{p^{\delta} \in \tilde{\mathbb{P}}_{x}} \frac{1}{\# \mathbb{Q}_{x}} \sum_{p^{\delta} \mid q} 1 .
$$


Assume $p \in \widetilde{\mathbb{P}}_{x}$ and $x$ is sufficiently large $\left(x \geq c_{7}\right)$. Using Lemma 3.1, we obtain

$$
\begin{aligned}
\frac{1}{\# \mathbb{Q}_{x}} \sum_{p \mid q} 1 & =\left(\# \mathbb{Q}_{x}\right)^{-1} \#\left\{\frac{m}{n}: \frac{m p}{n} \leq 1, n \leq x,(m p, n)=1\right\} \\
& =\left(\# \mathbb{Q}_{x}\right)^{-1} \#\left\{\frac{m}{n} \in \mathbb{Q}_{x}^{(0,1 / p]},(n, p)=1\right\} \\
& =\frac{1}{p+1}\left(1+O\left(\frac{\log x}{x}+\frac{p}{x}\right)\right)=\frac{1}{p+1}+O\left(\frac{\log x}{x}\right) .
\end{aligned}
$$

If $1 / p \in \widetilde{\mathbb{P}}_{x}$ and $x \geq c_{7}$, we have similarly from Lemma 3.1

$$
\frac{1}{\# \mathbb{Q}_{x}} \sum_{(1 / p) \mid q} 1=\frac{1}{p+1}\left(1+O\left(\frac{p \log (x / p)}{x}+\frac{1}{x}\right)\right)=\frac{1}{p+1}+O\left(\frac{\log x}{x}\right) .
$$

If $p^{\delta} \in \mathbb{P}_{x}$ and $p>\hat{x}:=x^{1-1 / \sqrt{\log x}}$, then

$$
\frac{1}{\# \mathbb{Q}_{x}} \sum_{p^{\delta} \mid q} 1 \leq \frac{1}{\# \mathbb{Q}_{x}} \sum_{n \leq x} \sum_{\substack{m \leq n \\ p^{\delta} \mid(m / n)}} 1 \ll \frac{1}{p}
$$

for $x \geq c_{7}$. Hence

$$
\begin{aligned}
\varphi(1, x) & =\sum_{\substack{p^{\delta} \tilde{\mathbb{P}}_{x} \\
p \leq \hat{x}}}\left(\frac{1}{p+1}+O\left(\frac{\log x}{x}\right)\right)+O\left(\sum_{\substack{p^{\delta} \in \mathbb{P}_{x} \\
p>\hat{x}}} \frac{1}{p}\right) \\
& =\sum_{p^{\delta} \in \tilde{\mathbb{P}}_{x}} \frac{1}{p+1}+O\left(\frac{\log x}{x} \sum_{p \leq \hat{x}} 1+\sum_{\substack{\hat{x}<p \leq x \\
p}} \frac{1}{p}\right) \\
& =\sum_{p^{\delta} \in \tilde{\mathbb{P}}_{x}} \frac{1}{p+1}+O\left(\log \left(1-\frac{1}{\sqrt{\log x}}\right)^{-1}+\mathrm{e}^{-\sqrt{\log x}}\right) .
\end{aligned}
$$

Finally $\varphi(1, x)=\sum_{\cdot p^{s} \in \widetilde{\mathbb{P}}_{x}}(p+1)^{-1}+\varepsilon(x)$.

Now let $l \geq 2$. It is easily seen that

$$
\varphi(l, x)=\sum^{*} \frac{1}{\# \mathbb{Q}_{x}} \sum_{\substack{q \in \mathbf{Q}_{x} \\ \delta_{1}^{\delta_{1}} \ldots p_{l} \mid q}} 1 .
$$

We split (6.3) into four parts and denote them by $\{\varphi(l, x)\}_{i}, i=1,2,3,4$. Into the first and second sums we include all summands for which $\delta_{1}, \delta_{2}, \ldots, \delta_{l}=1$ or -1 , respectively. The third sum

$$
\{\varphi(l, x)\}_{3}=\sum_{k=1}^{l-1}\left(\begin{array}{l}
l \\
k
\end{array}\right) \sum_{\substack{\delta_{1}, \ldots, \delta_{k}=1 \\
\delta_{k+1} \ldots \delta_{l}=-1 \\
p_{k+1} \cdots p_{l} \leq \hat{x}}}^{*} \frac{1}{\# \mathbb{Q}_{x}} \sum_{\substack{q \in \mathbb{Q}_{k} \\
\rho_{p} \cdots p_{k} \\
\rho_{k+1} \cdots p_{l}}} 1 .
$$


The fourth sum $\{\varphi(l, x)\}_{4}$ is constructed in the same manner as $\{\varphi(l, x)\}_{3}$ with the condition $p_{k+1} \cdots p_{l} \leq \hat{x}$ replaced by its opposite $p_{k+1} \cdots p_{l}>\hat{x}$.

If $\Delta_{x}\left(p_{1}^{\delta_{l}} \cdots p_{l}^{\delta_{l}}\right)=1$ for $p_{1}^{\delta_{1}} \cdots p_{l}^{\delta_{l}}$, we define

$$
P_{1}=P_{1}\left(p_{1}^{\delta_{1}} \cdots p_{l}^{\delta_{i}}\right)=\prod_{\substack{i=1 \\ \delta_{i}=1}}^{l} p_{i}, \quad P_{2}=P_{2}\left(p_{1}^{\delta_{1}} \cdots p_{l}^{\delta_{l}}\right)=\prod_{\substack{i=1 \\ \delta_{i}=-1}}^{l} p_{i}
$$

Let $p_{1}, p_{2}, \ldots, p_{l}$ be distinct prime numbers. It follows from Lemma 3.1 that, for $x$ sufficiently large $\left(x \geq c_{7}\right)$,

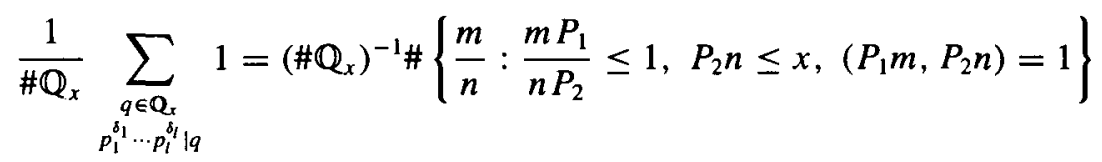

$$
\begin{aligned}
& =\left(\# \mathbb{Q}_{x}\right)^{-1} \#\left\{\frac{m}{n} \in \mathbb{Q}_{x}^{\left(0, P_{2} / P_{1}\right]},\left(m, P_{2}\right)=\left(n, P_{1}\right)=1\right\} \\
& =\prod_{p \mid P_{1} P_{2}} \frac{1}{p+1}\left(1+O_{l}\left(\frac{P_{2} \log x}{x}+\frac{P_{1}}{x}\right)\right) \\
& =\prod_{p \mid P_{1} P_{2}} \frac{1}{p+1}+O_{l}\left(\frac{\log x}{x P_{1}}+\frac{1}{x P_{2}}\right) \text {. }
\end{aligned}
$$

On the other hand, for each $p_{1}^{\delta_{1}} \cdots p_{l}^{\delta_{i}}=P_{1} / P_{2}$ we have

$$
\frac{1}{\# \mathbb{Q}_{x}} \sum_{\substack{q \in \mathbf{Q}_{x} \\\left(P_{1} / P_{2}\right) \mid q}} 1 \ll \frac{1}{x^{2}} \sum_{\substack{n \leq x \\ P_{2} \mid n}} \sum_{\substack{m \leq x \\ P_{1} \mid m}} 1 \leq \frac{1}{P_{1} P_{2}}
$$

for $x \geq c_{7}$.

Using condition (6.1), expression (6.4) and Landau's inequality (see, for example, [14]),

$$
\#\{m \leq x: \omega(n)=j\} \ll \frac{x}{(j-1) !} \frac{(\log \log x)^{j-1}}{\log x},
$$

where $j$ is a fixed natural number, we obtain

$$
\begin{aligned}
\{\varphi(l, x)\}_{1}= & \sum_{\delta_{1}, \ldots, \delta_{l}=1}^{*} \frac{\Delta_{x}\left(p_{1} \cdots p_{l}\right)}{\left(p_{1}+1\right) \cdots\left(p_{l}+1\right)} \\
& +O_{l}\left(\frac{\log x}{x} \sum_{\substack{\delta_{1}, \ldots, \delta_{l}=1 \\
p_{1} \cdots p_{l} \leq x}}^{*} \frac{1}{p_{1} \cdots p_{l}}+\frac{1}{x} \sum_{\substack{\delta_{1}, \ldots, \delta_{l}=1 \\
p_{1} \cdots p_{l} \leq x}}^{*} 1\right)
\end{aligned}
$$




$$
=\sum_{\delta_{1}, \ldots, \delta_{l}=1}^{*} \frac{\Delta_{x}\left(p_{1} \cdots p_{l}\right)}{\left(p_{1}+1\right) \cdots\left(p_{l}+1\right)}+\varepsilon_{l}(x),
$$

because the first remainder term is $O_{l}\left(x^{-1} \log x\left(\sum_{p^{\delta} \in \widetilde{\mathbb{P}}_{x}} 1 / p\right)^{l}\right)=O_{l}\left(x^{-1} \log x\right)$, and the second is $O_{l}\left(x^{-1} \#\{m \leq x: \omega(m)=l\}\right)=O_{l}\left((\log x)^{-1}(\log \log x)^{l-1}\right)$.

Similarly from (6.1) and (6.4)-(6.6), we have

(6.8) $\{\varphi(l, x)\}_{2}$

$$
\begin{aligned}
= & \sum_{\substack{\delta_{1}, \ldots, \delta_{l}=-1 \\
p_{1} \cdots p_{l} \leq \hat{x}}}^{*} \frac{\Delta_{x}\left(1 /\left(p_{1} \cdots p_{l}\right)\right)}{\left(p_{1}+1\right) \cdots\left(p_{l}+1\right)} \\
& \left.+O_{l}\left(\frac{1}{x_{\substack{\delta_{1}, \ldots, \delta_{l}=-1 \\
p_{1} \cdots p_{l} \leq \hat{x}}} \sum_{p_{1} \cdots p_{l}}}+\log x\right)+\sum_{\substack{\delta_{1}, \ldots, \delta_{l}=-1 \\
p_{1} \cdots p_{l}>\hat{x}}}^{*} \frac{\Delta_{x}\left(1 /\left(p_{1} \cdots p_{l}\right)\right)}{p_{1} \cdots p_{l}}\right) \\
= & \sum_{\delta_{1}, \ldots, \delta_{l}=-1}^{*} \frac{\Delta_{x}\left(1 /\left(p_{1} \cdots p_{l}\right)\right)}{\left(p_{1}+1\right) \cdots\left(p_{l}+1\right)}+\varepsilon_{l}(x)+O_{l}\left(W_{l}(x)\right),
\end{aligned}
$$

where

$$
W_{l}(x)=\sum_{\substack{\delta_{1}, \ldots, \delta_{l}=-1 \\ \hat{x}<p_{1} \cdots p_{l} \leq x}}^{*} \frac{1}{p_{1} \cdots p_{l}} .
$$

Using (6.1) and (6.4)-(6.6) again, we obtain

(6.9) $\{\varphi(l, x)\}_{3}$

$$
\begin{aligned}
& =\sum_{k=1}^{l-1}\left(\begin{array}{l}
l \\
k
\end{array}\right) \sum_{\substack{\delta_{1}, \ldots, \delta_{k}=1 \\
\delta_{k+1}, \ldots, \delta_{l}=-1 \\
p_{k+1} \cdots p_{l} \leq \hat{x}}}^{*} \frac{\Delta_{x}\left(\left(p_{1} \cdots p_{k}\right) /\left(p_{k+1} \cdots p_{l}\right)\right)}{\left(p_{1}+1\right) \cdots\left(p_{l}+1\right)} \\
& +O_{l}\left(\frac{1}{x} \sum_{k=1}^{l-1}\left(\begin{array}{l}
l \\
k
\end{array}\right) \sum_{\substack{\delta_{1}, \ldots, \delta_{k}=1 \\
\delta_{k+1, \ldots, \delta_{l}=-1} \\
p_{k+1} \cdots p_{l} \leq \hat{x}}}^{*} \Delta_{x}\left(\frac{p_{1} \cdots p_{k}}{p_{k+1} \cdots p_{l}}\right)\left(\frac{\log x}{p_{1} \cdots p_{k}}+\frac{1}{p_{k+1} \cdots p_{l}}\right)\right) \\
& =\sum_{k=1}^{l-1}\left(\begin{array}{l}
l \\
k
\end{array}\right) \sum_{\substack{\delta_{1}, \ldots, \delta_{k}=1 \\
\delta_{k+1}, \ldots, \delta_{l}=-1}}^{*} \frac{\Delta_{x}\left(\frac{p_{1} \cdots p_{k}}{p_{k+1} \cdots p_{l}}\right)}{\left(p_{1}+1\right) \cdots\left(p_{l}+1\right)}+\varepsilon_{l}(x)+O_{l}\left(\sum_{k=1}^{l-1} W_{l-k}(x)\right) .
\end{aligned}
$$

Finally, inequality (6.1) and estimate (6.5) imply that

$$
\{\varphi(l, x)\}_{4}=O_{l}\left(\sum_{k=1}^{l-1} W_{l-k}(x)\right) .
$$


Substituting (6.7)-(6.10) into (6.3), we can assert that

(6.11) $\varphi(l, x)=\sum^{*} \frac{\Delta_{x}\left(p_{1}^{\delta_{1}} p_{2}^{\delta_{2}} \cdots p_{l}^{\delta_{l}}\right)}{\left(p_{1}+1\right)\left(p_{2}+1\right) \cdots\left(p_{l}+1\right)}+\varepsilon_{l}(x)+O_{l}\left(\sum_{k=1}^{l-1} W_{k}(x)\right)$.

We have that

$$
W_{1}=\sum_{\substack{\left(1 / p_{1}\right) \in \tilde{\mathbb{P}}_{x} \\ \hat{x}<p \leq x}} \frac{1}{p} \leq \sum_{\hat{x}<p \leq x} \frac{1}{p}=\log \left(1-\frac{1}{\sqrt{\log x}}\right)^{-1}+O\left(\mathrm{e}^{-\sqrt{\log x}}\right) .
$$

Define $\hat{x}_{l}=x^{1-(\log x)^{-1 /(1+1)}}$ for natural number $l$. Applying (6.1) for each fixed $k \geq 2$, we obtain

$$
\begin{aligned}
& W_{k}(x) \\
& \leq \sum_{\substack{\delta_{1}, \ldots, \delta_{k-1}=-1 \\
p_{1} \cdots p_{k-1} \leq \hat{x}_{2}}}^{*(k-1)} \frac{1}{p_{1} \cdots p_{k-1}}\left(\log \left(1+\frac{\log (x / \hat{x})}{\log \left(\hat{x} /\left(p_{1} \cdots p_{k-1}\right)\right)}\right)\right. \\
& \left.+O\left(\frac{1}{\log \left(\hat{x} /\left(p_{1} \cdots p_{k-1}\right)\right.}\right)\right)+O_{k}\left(\sum_{\substack{\delta_{1} \ldots, \delta_{k-1}=-1 \\
\hat{x}_{2}<p_{1} \cdots p_{k-1} \leq x}}^{*(k-1)} \frac{1}{p_{1} \cdots p_{k-1}}\right) \\
& =O_{k}\left(\frac{1}{(\log x)^{1 / 6}}+\sum_{\substack{\delta_{1}, \ldots, \delta_{k-1}=-1 \\
\hat{x}_{2}<p_{1} \cdots p_{k-1} \leq x}}^{*(k-1)} \frac{1}{p_{1} \cdots p_{k-1}}\right) \\
& =O_{k}\left(\frac{1}{(\log x)^{1 / 6}}+\sum_{\substack{\delta_{1}, \ldots, \delta_{k-2}=-1 \\
p_{1} \cdots p_{k-2} \leq \hat{x}_{3}}}^{*(k-2)} \frac{1}{p_{1} \cdots p_{k-2}}\left(\log \left(1+\frac{\log \left(x / \hat{x}_{2}\right)}{\log \left(\hat{x}_{2} /\left(p_{1} \cdots p_{k-2}\right)\right)}\right)\right.\right. \\
& \left.\left.+O\left(\frac{1}{\log \left(\hat{x}_{2} /\left(p_{1} \cdots p_{k-2}\right)\right.}\right)\right)+\sum_{\substack{\delta_{1}, \ldots, \delta_{k-2}=-1 \\
\hat{x}_{3}<p_{1} \cdots p_{k-2} \leq x}}^{*(k-2)} \frac{1}{p_{1} \cdots p_{k-2}}\right) \\
& =O_{k}\left(\frac{1}{(\log x)^{1 / 12}}+\sum_{\substack{\delta_{1}, \ldots, \delta_{k-2=-1} \\
\hat{x}_{3}<p_{1} \cdots p_{k-2} \leq x}}^{*(k-2)} \frac{1}{p_{1} \cdots p_{k-2}}\right) \\
& =\cdots=O_{k}\left(\frac{1}{(\log x)^{1 /(k(k+1))}}+\sum_{\substack{\left.1 / p_{1}\right) \in \widetilde{\mathbb{P}}_{x} \\
\hat{x}_{k}<p_{1} \leq x}} \frac{1}{p_{1}}\right)=O_{k}\left(\frac{1}{(\log x)^{1 /(k(k+1))}}\right) .
\end{aligned}
$$


Equality (6.2) follows from (6.11). This completes the proof of Proposition 6.1.

\section{Proof of Theorem 1.1}

PROOF (Necessity). Let $v_{x}\left(f_{x}(q)<u\right) \Rightarrow P(X<u)$ for some integer-valued random variable $X$. It follows from Proposition 4.1 that

$$
\varphi(1, x)=\frac{1}{\# \mathbb{Q}_{x}} \sum_{q \in \mathbf{Q}_{x}} f_{x}(q) \ll 1 .
$$

Hence, according to Proposition 6.1, $\sum_{p^{b} \in \widetilde{\mathbb{F}}_{x}} 1 / p \leq c_{8}$, where $c_{8}$ depends on the structure of the limit law of $X$.

Using (6.5), we have for $x$ sufficiently large

$$
\nu_{x}\left(f_{x}(q)=k\right)=\frac{1}{k !} \sum^{* k} \frac{1}{\# \mathbb{Q}_{x}} \sum_{p_{1}^{\delta_{1}} \ldots p_{k}^{k_{k}} \mid q} 1 \ll \frac{1}{k !} \sum^{* k} \frac{1}{p_{1} \cdots p_{k}} \leq \frac{1}{k !}\left(\sum_{p^{\delta} \in \widetilde{\mathbb{P}}_{x}} \frac{1}{p}\right)^{k} .
$$

Since $\lim _{x \rightarrow \infty} v_{x}\left(f_{x}(q)=k\right)=P(X=k)$ for every $k=0,1,2, \ldots$, we obtain that $P(X=k) \ll c_{8}^{k} / k !, k \in \mathbb{N}$. Hence

$$
g_{l}=\sum_{k=l}^{\infty} k(k-1) \cdots(k-l+1) P(X=k) \ll \sum_{k=l}^{\infty} \frac{c_{8}}{(k-l) !}=c_{8}^{l} \mathrm{e}^{c_{8}}
$$

for each fixed natural $l$.

The necessity of the condition in Theorem 1.1 now follows from Propositions 5.1 and 6.1.

PROOF (Sufficiency). Let all the limits in the statement of Theorem 1.1 exist. Since

$$
\lim _{x \rightarrow \infty} \sum_{p^{\delta} \in \tilde{\mathbb{P}}_{x}} \frac{1}{p+1}=g_{1},
$$

we have that

$$
g_{l}=\lim _{x \rightarrow \infty} \sum^{*} \frac{\Delta_{x}\left(p_{1}^{\delta_{1}} \cdots p_{l}^{\delta_{l}}\right)}{\left(p_{1}+1\right) \cdots\left(p_{l}+1\right)} \leq\left(\lim _{x \rightarrow \infty} \sum_{p^{s} \in \widetilde{\mathbb{P}}_{x}} \frac{1}{p+1}\right)^{l}=g_{1}^{l} .
$$

Therefore the series $\sum_{l=1}^{\infty}\left(2^{l} g_{l} / l !\right)$ converges.

On the other hand, Proposition 6.1 implies that $\lim _{x \rightarrow \infty} \varphi(l, x)=g_{l}$ for each natural $l$.

The statement of Theorem 1.1 now follows from Proposition 5.1. 


\section{References}

[1] P. D. T. A. Elliott, Probabilistic number theory. I (Springer, Berlin, 1979).

[2] - Probabilistic number theory. $I$ (Springer, Berlin, 1980).

[3] G. Halász, 'On the distribution of additive arithmetical functions', Acta Arith. 27 (1975), 143-152.

[4] Z. Kryžius, 'Topics on arithmetic functions with rational arguments', in: Analytic and probabilistic methods in number theory (eds. F. Schweiger and E. Manstavičius), New trends in Probabability and Statistics 2 (VSP-TEV, Utrecht, Vilnius, 1992) pp. 235-249.

[5] J. Kubilius, Probabilistic methods in the theory of numbers, Translations of Mathematical Monographs 11 (Amer. Math. Soc., Providence, RI, 1964).

[6] I. Z. Ruzsa, 'The law of large numbers for additive functions', Studia Sci. Math. Hungar. 14 (1979), 247-253.

[7] _ 'Generalized moments of additive functions', J. Number Theory 18 (1984), 27-33.

[8] J. Šiaulys, 'Von Mises theorem in number theory', in: Analytic and probabilistic methods in number theory (eds. F. Schweiger and E. Manstavičius), New trends in Probabability and Statistics 2 (VSP-TEV, Utrecht, Vilnius, 1992) pp. 293-310.

[9] _ - 'Moments inequality for additive functions with rational argument', Fiz. Mat. Fak. Moksl. Semin. Darb. 6 (2003), 131-141.

[10] — 'The Halász's inequality for additive functions with rational argument', Liet. Mat. Rink. 44 (2004), 272-277, (in Russian).

[11] V. Stakènas, 'The additive functions and rational approximations', Lithuanian Math. J. 28 (1988), 260-272.

[12] — 'A sieve result for Farey fractions', Lithuanian Math. J. 39 (1999), 87-102.

[13] V. Stakènas and J. Šiaulys, 'Distribution of values of additive functions of a rational argument', Lithuanian Math. J. 28 (1988), 273-284.

[14] G. Tenenbaum, Introduction to analytic and probabilistic number theory, Cambridge Studies in Advanced Mathematics 46 (Cambridge University Press, Cambridge, 1995).

Department of Probability

Theory and Number Theory

Vilnius University

Naugarduko 24

03225 Vilnius

Lithuania

e-mail: jonas.siaulys@mif.vu.lt
Department of Mathematical Informatics

Vilnius University

Naugarduko 24

03225 Vilnius

Lithuania

e-mail: gediminas.stepanauskas@maf.vu.lt 